\title{
Recent Trends and Challenges in Virtual Reality
}

\author{
Sanjay S. \\ Department of CSE, \\ R.V College of Engineering, Bengaluru, \\ Karnataka, 560040, India
}

\author{
Girish Rao Salanke N. S. \\ Assistant Professor, Department of CSE \\ R.V College of Engineering, Bengaluru, \\ Karnataka, 560040, India
}

\begin{abstract}
This paper provides an overview of the Virtual Reality with emphasis on current trends of hardware and software technology, the processing and application issues. The first section of the paper presents a background of Virtual reality followed by the description and classification of the technology and the way the processing of those technologies takes place. Based on the observations from the current practice, the technologies are overviewed with an attempt to focus on areas it has evolved to nurture and trend the way users are characterized by it, followed by challenges of implementing new technology approaches in creating Virtual reality system. Finally, summarizing with issues related to engaging Virtual reality and on the future enhancement and developments
\end{abstract}

\section{Keywords}

Telepresence, Virtual Environment, Full Immersive Virtual Reality, Cyber sickness.

\section{INTRODUCTION}

Virtual reality typically means 'near-reality', which is what we experience as Humans [1] [2]. It has been about 52 years since Ivan Sutherland formally presented the world his vision of "Ultimate Display" imitating the real world in every sense. Then huge number of individual refreshing technologies supporting this had emerged. The term "Virtual Reality" then came into place formally by Jason Lanier, who was trying to summate Sutherland's vision in different concepts. This hyped many Researchers and Scholars to develop Technology and Algorithms to fulfil Sutherland's Dream for many years, which was the First wave of Virtual Reality. For Past 10 years, the second wave of Virtual Reality introduced new variety of input and display devices, but many of them failed due to possible reasons like poor technology failing to deliver required End-user experience, robustness and low-fidelity [3]. Every season, new successful experiments dealing with existing Problems on VR's Hardware and Software side are being exhibited.

This Paper mainly focuses on the aspects of current trends of technological approaches to problems on development of the Virtual Reality system. The current paper is based on an Internet and Publication survey depicting overall comparison of technological approaches by various Researchers to current trends of Virtual Reality system.

\section{TAXONOMY OF VIRTUAL REALITY ENVIRONMENT}

Virtual Reality is a system used to create a digital environment in which the user has the impression of being in that world with the ability to manipulate objects in that world and navigate through it. VR is capable of immersing the user in a computer generated world, be it, a city, a human body or your own mythological world. Using VR, you can map unexplored territory of the human imagination. A very important aspect of virtual reality is the environment in which it takes place because it determines the entire experience. So Virtual environment must be properly engineered with a view towards the aspect of Immersion. Human Beings often dealt for power to interact with the imaginary world with a mind-set of real world scenarios. This power of Immersion as well as interaction is called Telepresence. There are three levels of Telepresence which is often referred as Virtual Environment implementations:

1. Semi-Immersive Virtual Reality: Basically consist of large display system or projection systems with a wider field of view. With this concept, it can increase the feeling of immersion experienced by the user. This gives the user a partial feel of immersion but has a prominent benefit in educational applications as it allows simultaneous experience of the Virtual Environment that is not available with Full Immersive VR.

2. Full Immersive Virtual Reality: Basically consist of Head Mounted Display or Head- coupled display such as a Binocular Omni-Orientation Monitor or BOOM. This projects the most direct experience of Virtual Environment. It is the most widely known VR implementation. We are going to discuss the technologies involved in detail in the next section.

3. CAVE Immersive Virtual Reality: Basically consists of down projection floor, tracking sensors in the walls, Rear projection walls. This is like a combination of Semi Immersive and Full immersive VR where the user wearing a head mounted display (HMD) experiences Stereoscopy, displaying a three dimensional display. Here the user is shown two images, one for each eye for which the brain instigates and combines into one image.

4. Distributed/Collaborative Virtual Environment: Basically consist of a network. Here there will be simulated world running on several computers which are network connected and the users are able to share the same virtual world.

The most widely popular and rapidly growly VR is the Full Immersive VR, which the industries have caught, hold by the demand from Customer centric view point of Entertainment.

\section{TECHNOLOGY OF FULL IMMERSIVE VIRTUAL REALITY}

Full immersive VR has changed the way many users project their work. This is used in several areas like Automobile Industry reviewing a critical application through Telepresence via Head Mounted display and Data Glove, Gaming industry switching the way of gaming to Full Immersive VR via HMD that has scaled up a huge demand. So what is the technology approach used in current HMD and Data glove that are both 
Synced to specific application used by Several industry? First we discuss about the current technology approach for HMD.

A good HMD depends on a number of criteria like pixels, Retinal projection, Audio hardware, Refresh rate, Head tracking, Latency, Optics, Eye tracking. The second wave of VR launched two types of HMD; Console VR and Mobile VR. The console VR generally requires heavy graphics when toggling with headset. The system is used on a PC or a game console. The Oculus Rift is an example of a device where the Goggles have an integrated display and sensors. The games run on a separate system. Other desktop headsets include HTC's Vive and Sony's Morpheus for PlayStation. Mobile VR is a simple housing (device) for two lenses and a slot for your mobile phone. The phone's display is used to show the twin stereographic views. It has rotational head tracking, but it has no positional tracking. Cardboard also provides the user with the ability to click or tap its side to make selections in a game. The complexity of the imagery is limited because it uses your phone's processor for rendering the views on the phone display screen, therefore causing Slow-near field object response. The apparent shift position of the physical object with that of Actual position of the physical object rendering to perceived position of physical object can be very large or very small based on the device's display image plane. This causes a depth Perception error. These type of errors can also cause, what is known to be one of the VR sickness.

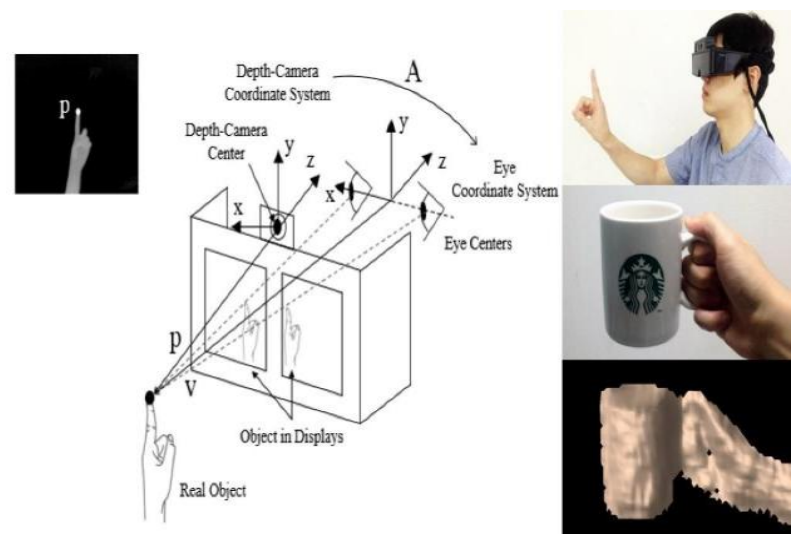

Figure 1. A Calibration Method for Optical See-through HMD with a Depth Camera

The proposed work as shown in Figure 1, solves the problem of Slow Near-field object response time. Here it takes the key merit of optical see-through head mounted display's accurate calibration, there by taking advantages of the affordable OSTHMDs, with depth cameras which are most appearing in commercial markets. So in order to correctly reflect your calibration process. In order to correctly reflect the user experience into the calibration process, this method demands a user wearing the HMD to repeatedly point at rendered virtual circles with their fingertips.

Secondly we discuss about the current technological approach in Data Glove [4]. A Data Glove as shown in Figure 2 is an interacting system that tactless fine-motion control. The FineMotion control involves the detection of movement of user's hand through sensors and then translates these motions into signals that can be used by Virtual hand. This allows us to interact normally with the objects in the Virtual world. This feedback is needed for effective immersiveness for applications like Gaming. Many types of data gloves have been produced for various purposes like Simulation, Air- writing and animation. The Flex sensors used are quite sensitive to immediate slight movement. They can trigger wrong motion on the projected area. There are mainly two popular approaches to solve this. One is to use distant camera that tracks the location of the fingers fitted with contact sensor, second is to use soft stretchable bending sensor that has Electron paramagnetic resonance (EPR).

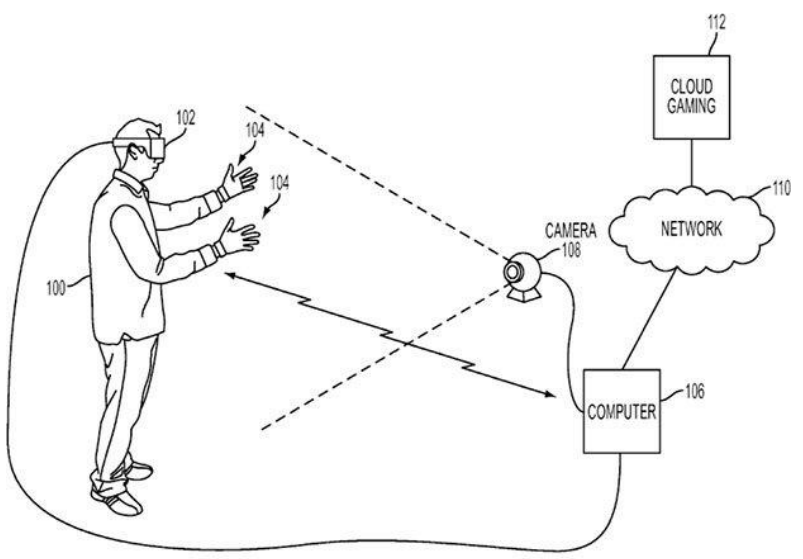

FIG. 1A

Figure 2. Data Glove Interaction, USPTO, Sony

This is the Sony's latest move on its Play Station Data glove. The user interface diagram shows a wireless glove containing wearable bracelets as shown in Figure 3 on the wrists of both hands. The bracelets each feature an attached LED light that can be used to identify glove location and orientation based on images captured by a PlayStation Camera. Similar to Sony's wand-like PlayStation Move controllers, the glove controllers' LED lights have adjustable colours, adjustable brightness intensities, and other controllable features. The bracelets each contain a small sensor data CPU and an entire variety of various sensors and buttons for user input.

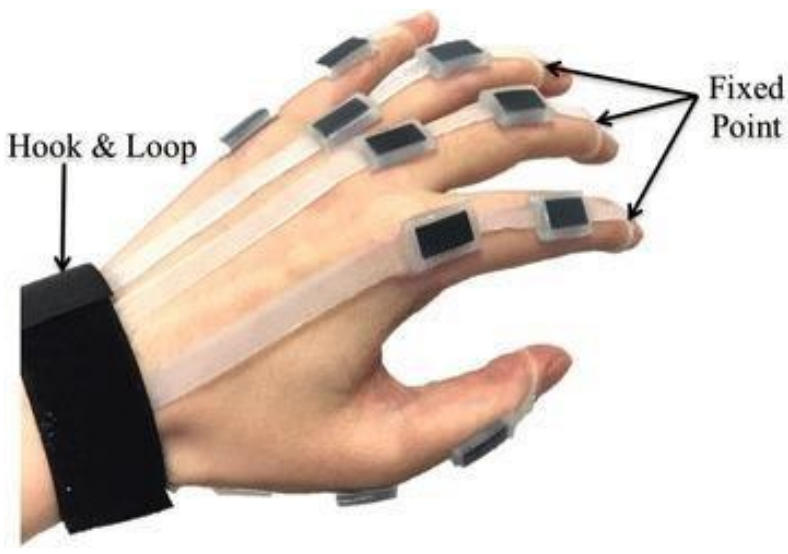

Figure 3. A soft stretchable bending sensor

This data glove mainly formulates the deformable nature of the human hand. Most sensors are non-stretchable, so there's need to accommodate stretching surface. The sensor's slipping on skin may are prone to cause errors. In this case, the sensors are insensitive to stretching, hence does not cause error. But this also faces few demerits where the plastic rubber sheets used are susceptible to repeatability, in the sense deformation property is held here. But this only causes minimal error during simulation. 


\section{TECHNOLOGY CHALLENGES OF VIRTUAL REALITY}

The VR technological challenges described in the field of consumer market deal with display quality and latency issues in order to decrease the health issues like cyber sickness. Most of the VR industry have to dealt with existing technologies being originally produced that are meant for other departments, and have to that the company stays in the business. In case of creation of Virtual world, it generally takes longer time for a powerful environment, the more powerful/realistic the environment, the more time it takes to complete it. Therefore it takes a team of developers more than a year to mask a real world accurately.

Much of this processing will require a more powerful CPU. This is the challenge of the Rendering Process. The biggest problem in Virtual reality technology is the clash between Rendering and Input process. Constant development have been happening to Input process of VR to keep up with the upheld task of rendering process with respect to Cost and ethical issues. Range of display quality among headsets have created a problem for the Rendering area that pulls down the Immersive experience.

\section{RISKS OF ENGAGING VIRTUAL REALITY AND ITS FUTURE DEVELOPMENTS}

Virtual Reality may have changed the way the world view things. It is gaining momentum as promising new technologies. Despite gaining proclamation regarding rise in VR in various fields, the risk associated with it are often ignored. Few of the risks associated are Cyber sickness / simulator sickness, Low-fidelity, Expensive and High-fidelity system. Few Virtual Environment development industry have taken this into consideration and are working towards developing a friendly VR set to the user. The following elements are considered during those development: ( Source: Doug Magyari, VR CNBC 2016 )

1. Lightweight comfort: Any head-worn equipment generally weighs less than $5 \mathrm{Kg}$, then they will feel uncomfortable after wearing them for short periods of time.

2. Large field of view (FoV): Humans generally have a stereoscopic field of view of 200 degrees and 60 degrees for peripheral vision. Restricting this FoV decreases the user's sense of presence in case Visual perception.

3. Natural GUI: A well-built user interface can facilitate organic experience. For example, Sony's PlayStation, Samsung Smart TV are having wristbased interface allowing users to control the system with gestures.

4. Natural eye optics: For $2 \mathrm{D} / 3 \mathrm{D}$ content, the VR industry should mimic their devices to real human eye optics in order to facilitate accurate result and comfortable feel. The headset makers need to solve the "Convergence conflict," to eliminate eye strain.

5. Combining AR/VR capabilities: The headsets should automatically control the translucency of real world. It should mitigate motion sickness, by anchoring themselves to real world, so it is necessary to adoptability.

6. Allowing for customization: Everyone are unique and should be given freedom to create their sense of device. Here the technology should adopt to their customized system with variable lenses, independent eye focus and inter-pupillary distance.

In the coming years, we will be engaged with virtual phones just like the one in Sci-fi movies like Star-trek, which will allow one person to see a 3D image of the other using VR techniques just like the Holographic talk as seen in the Sci-fi movies. Due to huge demand in Entertainment field, cinema industry are working to convert their usual 3D movies to VR. In India, the makers of a movie called "Baahubali-The Conclusion" are the first to produce it in VR. Therefore, the benefits of Virtual Reality will remain immeasurable.

\section{CONCLUSION}

As per the survey, you can see that the Sutherland's dream of Mimicking real world in all available sense is quite immeasurable. We have only achieved just about $30 \%$ of his dream. Though vast technological developments have been progressing, the feel of Near-Reality is still far from reach. But if you compare the First-wave of VR and Second-wave of $\mathrm{VR}$, it is seen that the goal of Ultimate display is converting from a science fiction to reality. The more powerful the computers, the more realistic graphic images be created to mitigate reality better. Virtual Reality's future depends on the existence large scale virtual environment development.

From 21st the mobile phones had changed our normal life, and now this VR is going to change the next generation who will be surrounded by Virtual Reality in all aspects of their life.

\section{REFERENCES}

[1] Christoph Anthes, Rubén Jesús García," State of the art of virtual reality technology", Aerospace Conference, IEEE 2016

[2] N.-N. Zhou, Y.-L. Deng, "Virtual reality: A state-of-theart survey", International Journal of Automation and Computing, vol. 6, no. 4, pp. 319-325, November 2009.

[3] Sharmistha Mandal," Brief Introduction of Virtual Reality \& its Challenges", International Journal of Scientific \& Engineering Research, Volume 4, Issue 4, April 2014.

[4] Piyush Kumar, Jyoti Verma and Shitala Prasad, "Hand Data Glove: A Wearable Real-Time Device for HumanComputer Interaction", International Journal of Advanced Science and Technology Vol. 43, June, 2013

[5] J. Logan Olson,David M. Krum, "A Design for a Smartphone-Based Head Mounted Display", IEEE Virtual Reality 2014.

[6] Jun, Hanseul, and Gunhee Kim. "A calibration method for optical see-through head-mounted displays with a depth camera”, IEEE Virtual Reality 2016.

[7] The Virtual Reality Society. www.vrs.org.uk/virtualreality-gear/head-mounted-displays. 